\title{
Two-Dimensional Open Microfluidic Devices by Tuning the Wettability on Patterned Superhydrophobic Polymeric Surface
}

\author{
Nuno M. Oliveira ${ }^{1,2}$, Ana I. Neto ${ }^{1,2}$, Wenlong Song ${ }^{1,2}$, and João F. Mano ${ }^{1,2 *}$ \\ ${ }^{1}$ 3B's Research Group, Department of Polymer Engineering, University of Minho, AvePark, Zona Industrial da Granja, \\ S. Cláudio do Barco, 4806-909 Caldas das Taipas, Guimarães, Portugal \\ ${ }^{2}$ Institute for Biotechnology and Bioengineering (IBB), Portuguese Government Associated Laboratory, $4800-019$ Guimarães, Portugal \\ Received June 2, 2010; accepted July 20, 2010; published online August 6, 2010
}

We present a simple and economical method to produce a potential open microfluidic polymeric device. Biomimetic superhydrophobic surfaces were prepared on polystyrene using a phase separation methodology. Patterned two-dimensional channels were imprinted on the superhydrophobic substrates by exposing the surface to plasma or UV-ozone radiation. The wettability of the channels could be precisely controlled between the superhydrophobic and superhydrophilic states by changing the exposure time. The ability of superhydrophilic paths to drive liquid flows in a horizontal position was found to be significantly higher than for the case of hydrophilic paths patterned onto smooth surfaces. (C) 2010 The Japan Society of Applied Physics

西

$\mathrm{n}$ the last few years, the research on superhydrophobic (SH) surfaces has aroused a high level of interest in the scientific community. These surfaces have a high water repellency, where water droplets can easily move around even when very small forces are applied. By definition $\mathrm{SH}$ surfaces present a contact angle (CA) higher than $150^{\circ}$ and a contactangle hysteresis lower than $10^{\circ} .^{1,2)}$ Extreme water repellent phenomena have already been found in nature, such as the classical example of the lotus leaf. These surfaces present a rough topography with a hierarchical organization at both the nano- and microscales. Many techniques have been used to reproduce such features to process artificial SH surface. ${ }^{1,2)}$ In our group, we have proposed the use of a phase separation method to produce $\mathrm{SH}$ polymeric substrates as model supports for cell attachment studies. ${ }^{3,4)}$ In this work, we demonstrate that $\mathrm{SH}$ polymeric substrates produce by this simple method can be used to fabricate open microfluidic devices.

In microfluidics, the wettability is very important to control the water movement at the microscale. In this context, SH surfaces could be used for the reduction of drag in microchannels ${ }^{5)}$ and to produce microvalves. ${ }^{6)}$ The microfluidic concept is usually associated with the use of closed microchannels. However the more recent concept of open microfluidics enables to guide a fluid in a planar or almost planar surface. There are two main approaches to construct open microfluidic systems: by chemically patterning flat surfaces to conceive distinct surface areas with different wettability; ${ }^{7)}$ and by using nonplanar surface topographies that can be fabricated by soft lithographic techniques $^{8)}$ and photolithographic methods. ${ }^{9)}$ Beyond the advantages of using conventional microfluidic devices, open microfluidics offer the possibility for micropipette manipulation into the device, due to the direct accessibility of the channels, and the cleaning is easier. ${ }^{8,10)}$

With this work we present a simple methodology to produce open microfluidic devices using a SH surface. Such open-air microfluidic substrates were produced by patterning hydrophilic/superhydrophilic paths on a $\mathrm{SH}$ polymeric surface using plasma or UV-ozone radiation. For the proof of concept, polystyrene (PS) films were treated in order to obtain $\mathrm{SH}$ properties based on a straightforward and inexpensive methodology described before. ${ }^{3)}$

*E-mail address: jmano@dep.uminho.pt

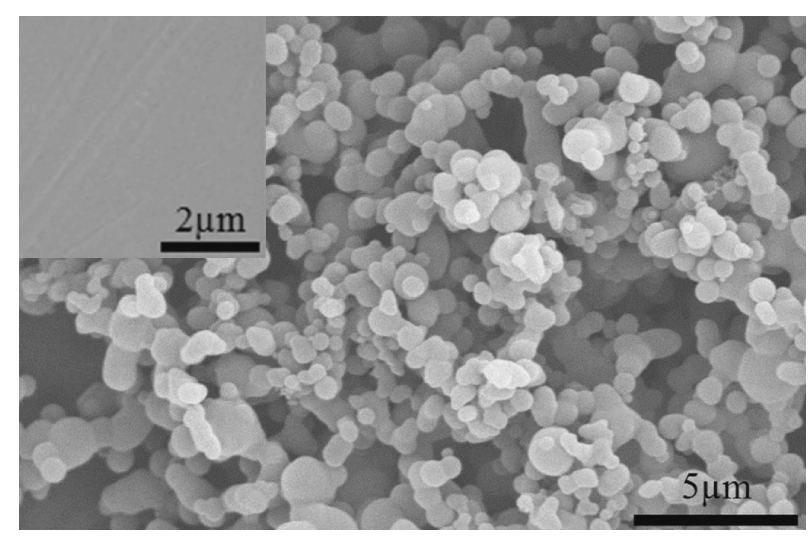

Fig. 1. Representative scanning electron microscopy image of the superhydrophobic rough PS surface. The inset image depicts the topography of the original smooth PS substrate.

A $70 \mathrm{mg} \cdot \mathrm{ml}^{-1}$ solution of PS (injection molding grade) in tetrahydrofuran was prepared, and then to each $2 \mathrm{ml}$ of this solution $1.3 \mathrm{ml}$ of ethanol was added. A few drops of this mixture were dipped onto a clean PS smooth substrate prepared by compression molding. After $10 \mathrm{~s}$ on air, the substrate with the mixture was immersed in ethanol for $1 \mathrm{~min}$. Afterwards the surface was dried under a nitrogen flow. We forced polymer precipitation with the immersion of the coated surface in a nonsolvent for the polymer (ethanol). The PS precipitation on the surface leads to formation of a rough surface due to the following mechanism: the mixture of a solvent and a nonsolvent of PS forms both poor and rich PS phases. ${ }^{11)}$ In the poor PS phase, polymer nuclei are formed by precipitation. The rich PS phase aggregates around these nuclei in order to decrease surface tension. ${ }^{12,13)}$ During polymer precipitation within the rich PS phase, a continuous deposition of spheres on the surface takes place. Figure 1 shows the representative microstructure development onto a PS substrate using this methodology. It is possible to observe sub-micrometer spheres that are agglomerated in larger micrometer structures.

The wettability of the surfaces was measured on a OCA $15+$ goniometer (DataPhysics) at room temperature (RT), where the static CA was measured by a sessile drop method with a $6 \mu \mathrm{l}$ water droplet. Using the same method, the contact angle hysteresis (CAH) was measured using a liquid 


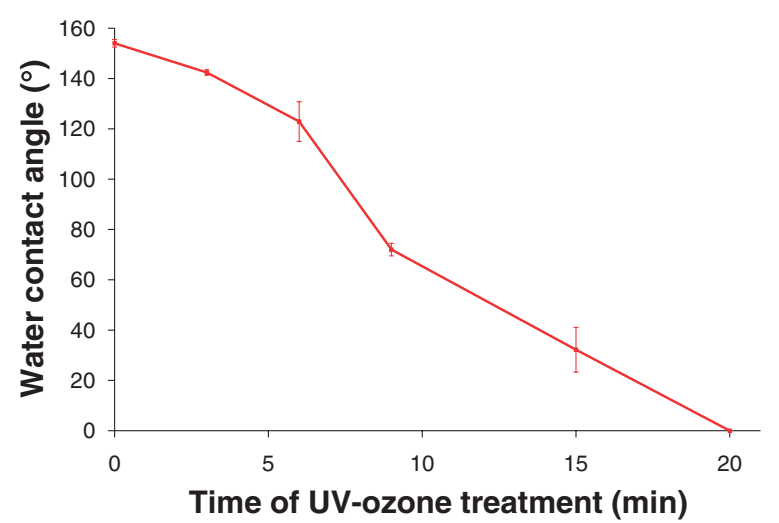

(a)

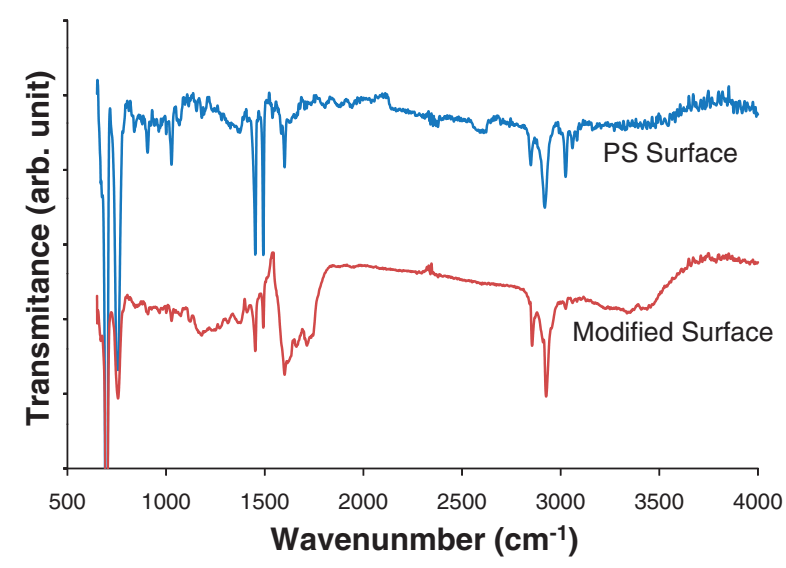

(b)

Fig. 2. (a) Influence of UV-Ozone treatment time on water CA of the $\mathrm{SH}$ surface. (b) FTIR-ATR spectra of SH PS surface before and after UVozone treatment for $20 \mathrm{~min}$.

injection rate of $0.3 \mu \mathrm{l} \cdot \mathrm{s}^{-1}$, altering the drop volume between 5 and $8 \mu \mathrm{l}$. The rough PS surface exhibited a CA of $154 \pm 1.5^{\circ}$ and $\mathrm{CAH}$ of $1.5 \pm 0.7^{\circ}$. The CA of the smooth surface was $86 \pm 2.7^{\circ}$.

Two theories are usually used to explain the superhydrophobic effect in rough surfaces. The Wenzel model predicts that roughness may be effective in increasing the CA of surfaces only if the CA of the smooth surface is higher than $90^{\circ}$, which was not observed for the PS substrate. ${ }^{14)}$ Cassie and Baxter postulated that the liquid does not intrude into the valleys of the rough surface and a fraction of the drop surface in contact with the substrate is suspended by air pockets. ${ }^{15-17)}$ In this case, the relation between the CA of the rough surface $\left(\theta^{*}\right)$ and the CA of the smooth surface $(\theta)$ is given by:

$$
\cos \theta^{*}=f(1+\cos \theta)-1
$$

where $f$ is the area fraction of the liquid-solid contact. From the CA measurements we can estimate that $f=0.1$.

To control the surface wettability we used a UV-ozone treatment with a PROCLEANER 220 (BioForce Nanoscience). This equipment generates light with two wavelengths: 185 and $254 \mathrm{~nm}$. By increasing the exposure time, the CA of the $\mathrm{SH}$ surface decreases until to the superhydrophilic regime [see Fig. 2(a)]. The results show that the wettability of the PS rough surface could be precisely controlled by changing the treatment time. The increase of wettability was the result

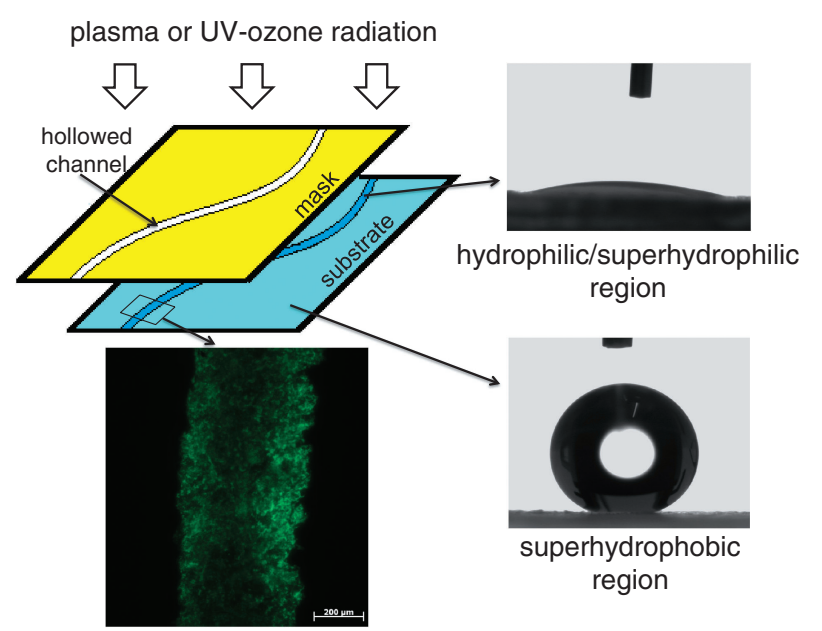

Fig. 3. Schematic representation of the methodology employed for the patterning of the surfaces, where hydrophilic/superhydrophilic channellike regions can be imprinted onto superhydrophobic surfaces. The fluorescent microscopy image highlights the section of a superhydrophilic channel on the SH surface after depositing and drying a FITC solution along the channel.

of surface oxidation where carboxyl and hydroxyl functional groups are usually formed. ${ }^{18,19)}$ These chemical changes were confirmed by Fourier transform infrared-attenuated total reflectance (FTIR-ATR) analysis, using a Spectrum 100 FT-IR Spectrometer from PerkinElmer [see Fig. 2(b)]. Two main bands can be detected in the modified surface that are not present in the initial PS substrate: one placed between 1550 and $1870 \mathrm{~cm}^{-1}$ and the other between 3200 and $3650 \mathrm{~cm}^{-1}$. The first absorption band is associated with the presence of carbonyl groups, like aldehyde, ketone or carboxilyc acid. The second band can be associated to hydroxyl groups. ${ }^{20)}$ Davidson et al. also reported a band for carbonyl groups in PS for cell culture dishes after UV-ozone treatment. ${ }^{18)}$ Vicente et al. also detected the two bands presented in this work, after oxidation of PS particles, using vacuum-UV irradiation in the presence of oxygen and/or ozone. ${ }^{19)}$

To pattern hydrophilic regions on the SH surface, we used masks of polymeric sheets in which open lines were cut to produce channel-shaped features. Such masks were placed over the SH substrate. During UV-ozone irradiation only the exposed regions of the mask were oxidized (see Fig. 3). Using different exposure time, channels with different wettabilities were obtained. In the examples presented here, the surfaces were exposed to UV-ozone for $20 \mathrm{~min}$ using a mask with open channels of $\approx 500 \mu \mathrm{m}$ width. After this process, we obtained superhydrophilic paths imprinted on the SH surface. The fluorescence optical microscopy image of Fig. 3 shows the shape of a superhydrophilic channel section. To obtain this fluorescent fingerprint, we made a fluorescein isothiocyanate (FITC) flow pass along the channel and then we let the remaining FITC solution in the surface dry at RT. The contour of the channel exhibits some irregularities at the micrometer level that could be caused by a not perfect contact between the hollowed mask and the surface.

The capability of the channels to withstand different water flows was tested using a patterned channel with a "L" shape (see Fig. 4). For comparison, PS smooth surfaces patterned 


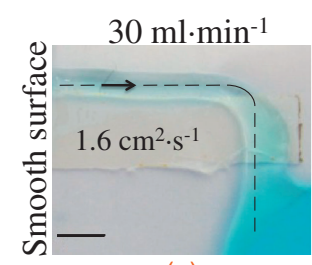

(a)

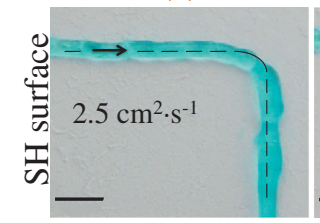

(d)

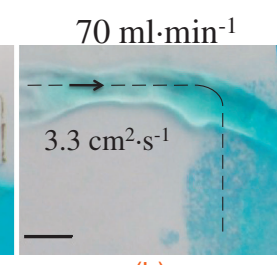

(b)

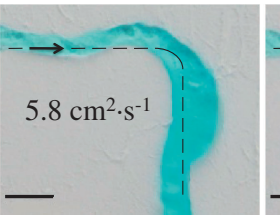

(e)

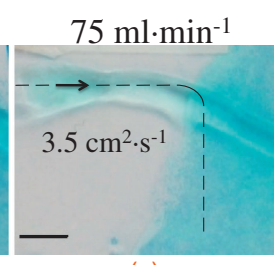

(c)

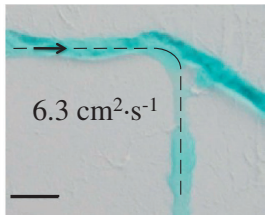

(f)
Fig. 4. Streams of colored water passing with different flow rates (in $\mathrm{ml} \cdot \mathrm{min}^{-1}$ ) on channels with a "L" shape patterned on smooth (above) and $\mathrm{SH}$ surfaces (below). The values inside the images correspond to the volumetric flow rate normalized by the width of the liquid column. The scale bars are $5 \mathrm{~mm}$.

in the same way as the $\mathrm{SH}$ surfaces were used. In the patterned smooth surfaces, the channels presented a CA of $13 \pm 0.6^{\circ}$. Figure 4 shows the differences between a flow on a smooth surface [(a)-(c)] and a SH surface [(d)-(f)] with the same patterned paths. In the SH surfaces, this paths could stand values of the order of tens of $\mathrm{ml} \cdot \mathrm{min}^{-1}$. Figure 4(d) shows that a flow of $30 \mathrm{ml} \cdot \mathrm{min}^{-1}$ dispensed in such patterned surface follows the superhydrophilic path, the substrate stood in a horizontal position. Only for a $70 \mathrm{ml} \cdot \mathrm{min}^{-1}$ stream some instability could be seen with some accumulation of liquid after the change of the direction [see Fig. 4(e)]. For $75 \mathrm{ml} \cdot \mathrm{min}^{-1}$, the water flow cannot follow the patterned channel, because the flow momentum overcomes the forces associated with the wettability contrast and the ability to retain the flow is disrupted. Regarding the smooth surface, it was not possible to control the flow of water along the hydrophilic path. To do the comparison between the different situations presented here, we normalize the flow value with the width of the flow on the surface. In other words, we divided the volumetric flow rate value by the average width of the respectively flow column, which was measured from the obtained images. These normalized values are showed in the respectively photographs of Figs. 4 and 5 .

In the patterned channels on SH surfaces, the streams presented an irregular shape which can be explained by a surface tension driven instability. Koplik et al. showed that if the CA between the stream and the surface is larger than $90^{\circ}$, the flow presents a similar shape to the RayleighPlateau instability. ${ }^{21)}$ Along the time, theses irregularities are stable with the flow.

As showed in Fig. 5, patterns with other shapes can be produced on the surface allowing the conception of more complex open microfluidic systems. Figure 5(a) shows that a flow of $50 \mathrm{ml} \cdot \mathrm{min}^{-1}$ could follow a superhydrophilic path with a "S" shape. Figure 5(b) shows that the two liquids with different colors, flowing initially with a rate of $8 \mathrm{ml} \cdot \mathrm{min}^{-1}$, maintain some separation in the initially common channel after the contact region, in a surface with a patterned "Y" shape path. Along of the channel the merging of the two liquids occurs gradually.

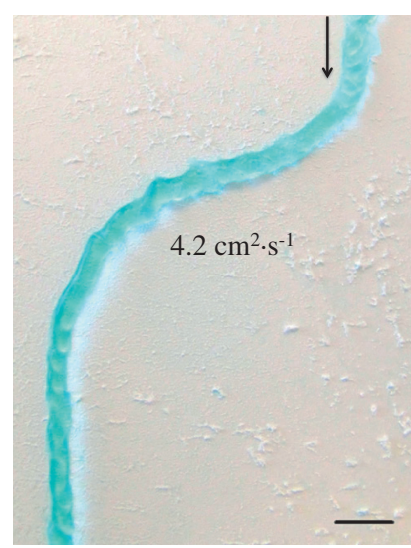

(a)

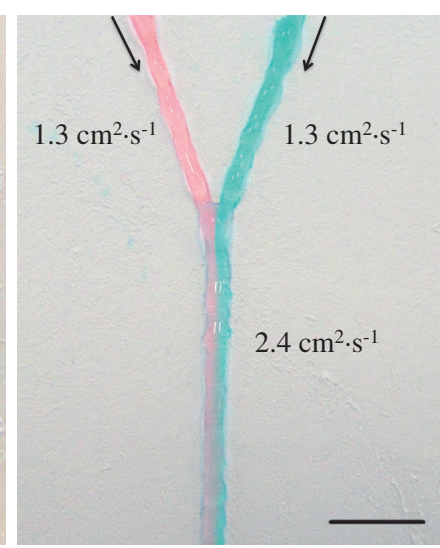

(b)
Fig. 5. Two examples of patterned $\mathrm{SH}$ surfaces with $\mathrm{S}$ shape (a) and a $Y$ junction in which the interface between two liquids is showed (b). The values inside the images correspond to the volumetric flow rate normalized by the width of the liquid column. The scale bars are $5 \mathrm{~mm}$.

In conclusion, we have developed an easy, inexpensive and fast way to fabricate open microfluidic polymeric devices. We envisage that more complex and perfect channels with controlled wettability could be patterned on such substrates and this technology has the potential to be used in a variety of applications, including in bioengineering and biomedicine.

1) P. Roach, N. J. Shirtcliffe, and M. I. Newton: Soft Matter 4 (2008) 224.

2) X. Zang, F. Shi, J. Niu, Y. Jiang, and Z. Wang: J. Mater. Chem. 18 (2008) 621.

3) W. Song, D. D. Veiga, C. A. Custódio, and J. F. Mano: Adv. Mater. 21 (2009) 1830

4) N. M. Alves, J. Shi, E. Oramas, J. L. Santos, H. Tomás, and J. F. Mano: J. Biomed. Mater. Res., Part A 91A (2009) 480.

5) C.-H. Choi and C.-J. Kim: Phys. Rev. Lett. 96 (2006) 066001.

6) C. Lu, Y. Xie, Y. Yang, M. M.-C. Cheng, C.-G. Koh, Y. Bai, L. J. Lee, and Y.-J. Juang: Anal. Chem. 79 (2007) 994.

7) H. Gau, S. Herminghaus, P. Lenz, and R. Lipowsky: Science 283 (1999) 46.

8) C.-H. Hsu, C. Chen, and A. Folch: Lab Chip 4 (2004) 420.

9) R. Seemann, M. Brinkmann, E. J. Kramer, F. F. Lange, and R. Lipowsky: Proc. Natl. Acad. Sci. U.S.A. 102 (2005) 1848.

10) L. Zhai, M. C. Berg, F. Ç. Cebeci, Y. Kim, J. M. Milwid, M. F. Rubner, and R. E. Cohen: Nano Lett. 6 (2006) 1213.

11) H. Y. Erbil, A. L. Demirel, Y. Avci, and O. Mert: Science 299 (2003) 1377.

12) Z. Yuan, H. Chen, J. Tang, X. Chen, D. Zhao, and Z. Wang: Surf. Coat. Technol. 201 (2007) 7138.

13) N. Zhao, J. Xu, Q. Xie, L. Weng, X. Guo, X. Zhang, and L. Shi: Macromol. Rapid Commun. 26 (2005) 1075.

14) R. N. Wenzel: Ind. Eng. Chem. 28 (1936) 988.

15) A. B. D. Cassie and S. Baxter: Trans. Faraday Soc. 40 (1944) 546.

16) A. Marmur: Langmuir 20 (2004) 3517.

17) S. Wang and L. Jiang: Adv. Mater. 19 (2007) 3423.

18) M. R. Davidson, S. A. Mitchell, and R. H. Bradley: Surf. Sci. 581 (2005) 169.

19) J. S. Vicente, J. L. Gejo, S. Rothenbacher, S. Sarojiniamma, E. Gogritchiani, M. Wörner, G. Kasper, and A. M. Braun: Photochem. Photobiol. Sci. 8 (2009) 944.

20) J. Coates: in Encyclopedia of Analytical Chemistry, ed. R. A. Meyers (Wiley, Chichester, U.K., 2000) p. 10815.

21) J. Koplik, T. S. Lo, M. Rauscher, and S. Dietrich: Phys. Fluids 18 (2006) 032104. 